\title{
Cord blood metabolic markers are strong mediators of the effect of maternal adiposity on fetal growth in pregnancies across the glucose tolerance spectrum: the PANDORA study
}

\author{
I-Lynn Lee ${ }^{1}$ • Elizabeth L. M. Barr ${ }^{1,2} \cdot$ Danielle Longmore $^{1} \cdot$ Federica Barzi $^{1} \cdot$ Alex D. H. Brown ${ }^{3,4} \cdot$ Christine Connors $^{5}$. \\ Jacqueline A. Boyle ${ }^{6}$. Marie Kirkwood ${ }^{1}$. Vanya Hampton ${ }^{1} \cdot$ Michael Lynch $^{7}$. Zhong X. Lu $^{8,9} \cdot$ Kerin O'Dea $^{10}$.

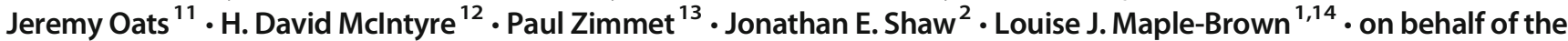 \\ PANDORA study team
}

Received: 2 September 2019 / Accepted: 23 October 2019 / Published online: 8 January 2020

(C) Springer-Verlag GmbH Germany, part of Springer Nature 2020

\begin{abstract}
Aims/hypothesis We aimed to assess associations between cord blood metabolic markers and fetal overgrowth, and whether cord markers mediated the impact of maternal adiposity on neonatal anthropometric outcomes among children born to Indigenous and Non-Indigenous Australian women with normal glucose tolerance (NGT), gestational diabetes mellitus (GDM) and pregestational type 2 diabetes mellitus.

Methods From the Pregnancy and Neonatal Outcomes in Remote Australia (PANDORA) study, an observational cohort of 1135 mother-baby pairs, venous cord blood was available for 645 singleton babies (49\% Indigenous Australian) of women with NGT $(n=129), \operatorname{GDM}(n=419)$ and type 2 diabetes $(n=97)$. Cord glucose, triacylglycerol, HDL-cholesterol, C-reactive protein (CRP) and C-peptide were measured. Multivariable logistic and linear regression were used to assess the associations between cord blood metabolic markers and the outcomes of birthweight $z$ score, sum of skinfold thickness (SSF), being large for gestational age (LGA) and percentage of body fat. Pathway analysis assessed whether cord markers mediated the associations between maternal and neonatal adiposity.

Results Elevated cord C-peptide was significantly associated with increasing birthweight $z$ score $(\beta 0.57$ [95\% CI 0.42, 0.71]), SSF ( $\beta 0.83$ [95\% CI 0.41, 1.25]), percentage of body fat ( $\beta 1.20$ [95\% CI 0.69, 1.71]) and risk for LGA [OR 3.14 [95\% CI 2.11, 4.68]), after adjusting for age, ethnicity and diabetes type. Cord triacylglycerol was negatively associated with birthweight $z$ score for Indigenous Australian women only. No associations between cord glucose, HDL-cholesterol and CRP $>0.3 \mathrm{mg} / \mathrm{l}(2.9 \mathrm{nmol} / \mathrm{l})$ with neonatal outcomes were observed. C-peptide mediated $18 \%(95 \%$ CI 13, 36) of the association of maternal BMI with LGA and $11 \%(95 \%$ CI 8,17$)$ of the association with per cent neonatal fat.
\end{abstract}

Louise J. Maple-Brown

Louise.Maple-Brown@menzies.edu.au

$1 \quad$ Wellbeing and Preventable Chronic Disease Division, Menzies School of Health Research, Charles Darwin University, PO Box 41096, Casuarina, NT 0811, Australia

2 Baker Heart and Diabetes Institute, Melbourne, VIC, Australia

3 South Australian Health and Medical Research Institute, Adelaide, SA, Australia

4 Faculty of Health and Medical Sciences, the University of Adelaide, Adelaide, SA, Australia

5 Northern Territory Department of Health, Darwin, NT, Australia

6 Monash Centre for Health Research and Implementation, School of Public Health and Preventative Medicine, Monash University, Clayton, VIC, Australia
7 Pathology Network, Top End Health and Hospital Services, Darwin, NT, Australia

8 Monash Pathology, Monash Health, Melbourne, VIC, Australia

9 Department of Medicine, Monash University, Melbourne, VIC, Australia

10 School of Health Sciences, University of South Australia, Adelaide, SA, Australia

11 Melbourne School of Population and Global Health, University of Melbourne, Melbourne, VIC, Australia

12 Mater Medical Research Institute, University of Queensland, Brisbane, QLD, Australia

13 Department of Diabetes, Central Clinical School, Monash University, Melbourne, VIC, Australia

14 Division of Medicine, Royal Darwin Hospital, Darwin, NT, Australia 


\section{Research in context}

\section{What is already known about this subject?}

- Cord C-peptide is strongly associated with neonatal adiposity in women with gestational diabetes but few studies include women with type 2 diabetes in pregnancy

\section{What is the key question?}

- What is the impact of maternal BMI, diabetes type, ethnicity and fetal sex on the relationships between cord blood biomarkers (glucose, triacylglycerol and C-peptide) and neonatal adiposity outcomes among women across the spectrum of glucose tolerance in pregnancy (normal glucose tolerance, gestational diabetes or type 2 diabetes)?

\section{What are the new findings?}

- $\quad$ Cord C-peptide was strongly associated with neonatal adiposity after adjusting for covariates

- Cord blood triacylglycerol was negatively associated with birthweight z score (significant for Indigenous Australian women only) after adjusting for covariates

- Cord blood C-peptide mediated approximately $18 \%$ of the contribution of maternal BMI to infants being born large for gestational age and $11 \%$ to percentage neonatal body fat

How might this impact on clinical practice in the foreseeable future?

- Our findings support the importance of population health interventions to reduce maternal BMI pre-pregnancy and to limit gestational weight gain among women with and without type 2 diabetes, in order to improve neonatal adiposity outcomes

Conclusions/interpretation Cord blood C-peptide is an important mediator of the association between maternal and infant adiposity, across the spectrum of maternal glucose tolerance.

Keywords Cord blood $\cdot$ Diabetes in pregnancy $\cdot$ Fetal hyperinsulinaemia $\cdot$ Gestational diabetes $\cdot$ Neonatal adiposity $\cdot$ Neonatal fat mass $\cdot$ Type 2 diabetes

$\begin{array}{ll}\text { Abbreviations } & \\ \text { CRP } & \text { C-reactive protein } \\ \text { DIP } & \text { Diabetes in pregnancy } \\ \text { GDM } & \text { Gestational diabetes mellitus } \\ \text { GWG } & \text { Gestational weight gain } \\ \text { HAPO } & \text { Hyperglycemia and Adverse Pregnancy } \\ & \text { Outcome } \\ \text { LGA } & \text { Large for gestational age } \\ \text { LPL } & \text { Lipoprotein lipase } \\ \text { NGT } & \text { Normal glucose tolerance } \\ \text { PANDORA } & \text { Pregnancy and Neonatal Outcomes } \\ & \text { in Remote Australia } \\ \text { ROLO } & \text { Randomised Control Trial of Low Glycaemic } \\ & \text { Index in Pregnancy } \\ \text { SSF } & \text { Sum of skinfold thickness }\end{array}$

\section{Introduction}

The worldwide epidemic of type 2 diabetes mellitus and increasing prevalence among younger people has led to a rising prevalence of type 2 diabetes in pregnancy, which is associated with adverse perinatal outcomes [1,2]. The prevalence of type 2 diabetes has overtaken that of type 1 diabetes in pregnancy in many populations [3-5]. Although advances in the clinical management of hyperglycaemia during pregnancy have resulted in reduced rates of large for gestational age (LGA) [6], normalisation of other neonatal outcomes including neonatal hypoglycaemia, hyperinsulinaemia and neonatal fat mass remain elusive [6-8]. This may be partly explained by the fetal glucose steal phenomenon whereby fetal hyperinsulinaemia early in pregnancies complicated by diabetes results in persistent placental glucose transfers from the mother to the infant throughout the pregnancy, despite improved maternal blood glucose levels later in pregnancy $[9,10]$. In addition to hyperglycaemia, elevated maternal lipids may also be important determinants of fetal growth [11-14]. Thus, the intrauterine environment is an important antecedent of increased future risk of obesity in offspring, and the transgenerational cycle of obesity and type 2 diabetes, such as that reported among Native Americans [15, 16].

Metabolic markers measured in neonatal cord blood provide insights into the placental transfer of these nutrients. Several 
studies show that abnormal cord blood metabolic marker levels are associated with fetal adiposity in women with normal glucose tolerance (NGT) and gestational diabetes mellitus (GDM) [17-19]. In normoglycaemic women both maternal glucose concentrations [20] and cord glucose [20] have been shown to influence fetal growth and neonatal adiposity. Lower cord blood levels of adiponectin and C-reactive protein (CRP), markers of glucose and NEFA metabolism and inflammation, respectively, were associated with a higher neonatal adiposity in the Hyperglycemia and Adverse Pregnancy Outcome (HAPO) study, which excluded women with type 2 diabetes [17]. In the Randomised Control Trial of Low Glycaemic Index in Pregnancy (ROLO) study with normoglycaemic women, higher cord blood leptin was associated with greater neonatal adiposity independent of sex, maternal BMI and gestational age but fetal C-peptide was not significant after adjustments [18]. ROLO also reported that lower cord blood triacylglycerol was associated with higher birthweight [21].

Few studies have assessed the role of these markers specifically in women with pregestational type 2 diabetes [22, 23], which is associated with greater risk of adverse pregnancy outcomes due to the exposure of the fetus to blood glucose earlier in the pregnancy [2]. A small study from New Zealand, comprising of Pacific Island women, reported increasing cord leptin and insulin concentrations across offspring groups from women with normal blood glucose levels $(n=95), \operatorname{GDM}(n=138)$ and type 2 diabetes $(n=39)$. In addition, correlations between cord insulin and birthweight $z$ score were stronger in women with type 2 diabetes, indicating that type 2 diabetes conferred a more severe intrauterine metabolic environment than that arising from lesser degrees of hyperglycaemia [23]. A study of Hispanic and Native American women found that cord leptin levels were associated with maternal hyperglycaemia; however, women with GDM and type 2 diabetes were grouped together [22].

Similar to other indigenous populations, Indigenous Australian women are at very high risk of obesity and type 2 diabetes and are ten times more likely to have pregestational type 2 diabetes than non-Indigenous Australian women [24]. The Pregnancy and Neonatal Diabetes Outcomes in Remote Australia (PANDORA) study is a prospective observational study of 1135 Indigenous and non-Indigenous Australian women and 1170 children born to women with NGT, GDM and type 2 diabetes [25]. The PANDORA study has previously reported on the strong associations between type 2 diabetes and poorer birth outcomes [17] and neonatal adiposity [26]. In this analysis, we extend our previous work and assess the following: (1) the impact of maternal BMI, diabetes type, ethnicity and fetal sex on the relationships between cord blood biomarkers (glucose, triacylglycerol and C-peptide) and neonatal adiposity outcomes (birthweight $z$ score, sum of skinfold thickness [SSF], LGA and percentage of body fat) among Indigenous and non-Indigenous Australian women; and (2) whether cord markers mediated the impact of maternal adiposity on neonatal anthropometric outcomes.

\section{Methods}

Study design and participants The PANDORA study is a prospective observational study conducted in the Northern Territory, Australia [25]. Baseline assessments were undertaken between November 2011 and February 2017. The PANDORA study sits within the Diabetes across the Lifecourse: Northern Australia Partnership, a partnership between researchers, policy makers and service providers to improve systems and services for all women with hyperglycaemia in pregnancy $[27,28]$. Women diagnosed with hyperglycaemia in pregnancy were invited from the Northern Territory Diabetes in Pregnancy Clinical Register to participate in PANDORA. Demographics, antenatal and peripartum information for 1171 babies were collected. Of these, a convenience sample of women without hyperglycaemia in pregnancy $(n=$ 235) was recruited from antenatal and midwifery clinics and was representative of women who birthed in the Northern Territory in terms of age and geographic location, compared with the Northern Territory Midwife Data Collection database (a census of all Northern Territory births) in 2013. For this analysis, we excluded babies (and their mothers) as follows: (1) babies from mothers with type 1 diabetes $(n=19)$; (2) twins ( $n=16$ [32 babies]); and (3) those who did not have cord blood collected $(n=475)$.

Ethics The study was approved by the Human Research Ethics Committee of the Northern Territory Department of Health and Menzies School of Health Research and the Central Australian Human Research Ethics Committee, and participants provided written informed consent.

Diagnosis of maternal diabetes The GDM diagnostic guidelines changed in Australia and worldwide during the course of the study. Between 2012 and 2014, there was a gradual increase in implementation of new guidelines throughout the Northern Territory. Therefore, women with GDM were diagnosed by either the 1999 Australian Diabetes in Pregnancy Society (ADIPS) guideline [29], or a universal 75 g OGTT with revised glucose cut-off values recommended by the International Association of the Diabetes and Pregnancy Study Group (IADPSG) [30] and the WHO [31]. Diabetes in pregnancy (DIP) was determined if OGTT or $\mathrm{HbA}_{1 \mathrm{c}}$ results were consistent with a diagnosis of type 2 diabetes (fasting glucose $\geq 7.0 \mathrm{mmol} / 1$, and/or $2 \mathrm{~h}$ glucose $\geq 11.1 \mathrm{mmol} / 1$, and/or $\mathrm{HbA}_{1 \mathrm{c}} \geq 47.5 \mathrm{mmol} / \mathrm{mol}$ [6.5\%]) but not diagnosed prior to pregnancy. NGT was determined by OGTT. 
Maternal measures Maternal characteristics assessed included maternal age, ethnicity, any smoking in pregnancy (yes/no), BMI (from first measured weight and height during pregnancy) and gestational weight gain (calculated as the difference between the third trimester weight closest to delivery and first measured weight). Pre-eclampsia was defined as per International Society for Study of Hypertension in Pregnancy guidelines [32]. Chronic hypertension was defined as hypertension diagnosed prior to pregnancy.

Neonatal outcome measures Birth characteristics assessed included sex and gestational age at birth. Neonatal measures collected included gestational age at birth, sex, birthweight, length, sum of three skinfold (flank, subscapular and triceps) thicknesses (mm) and percentage of body fat. Detailed anthropometric measures have been described elsewhere [26]. Birthweight SD $z$ scores were adjusted for sex and gestational age at birth using Australian normative data [33] and LGA was defined as a birthweight greater than the 90th centile according to these data. Per cent neonatal fat was calculated from the fat mass calculation outlined below [34].

Body fat mass was determined according to the following validated equation: Fat mass $=1000$ [0.39055 (birthweight [g]/1000)+0.0453 (flank skinfold thickness [mm]) -0.03237 (length [cm]) +0.54657$]$ [34-38]. Two neonates had negative fat mass according to the equation, and a fat mass of $0 \mathrm{~g}$ was substituted. Percentage of body fat was determined by calculating total body fat as a percentage of birthweight. A sensitivity analysis that excluded the two neonates with a $0 \%$ fat mass showed that the results were largely unchanged.

Venous cord blood was collected from 645 singleton babies (NGT $n=129$, GDM/DIP $n=419$, type 2 diabetes $n=97$ ) at delivery for analysis of glucose, C-peptide, HDL-cholesterol and triacylglycerol. The specimen was obtained by free drainage (free flow) of cord blood or drawn by needle aspiration (puncture) from a clamped segment of an umbilical vein. The specimen was stored in a styrofoam cooler for transport to the hospital's laboratory. Collection of cord blood was not feasible for all participants due to the unpredictable nature of birth and delivery and the need to prioritise clinical care. Cord blood glucose, HDL-cholesterol and triacylglycerol were measured using Vitros Fusion 5.1 (Ortho-Clinical Diagnostics, Raritan, NJ, USA). Cord blood C-peptide was measured using the electrochemiluminescence immunoassay on a cobas e602 (Roche Diagnostics, Mannheim, Germany) and CRP on a cobas c701 (Roche Diagnostics).

Statistical methods Data were analysed using Stata 15.0 (Stata, College Station, TX, USA). Maternal and neonatal characteristics were described according to three maternal hyperglycaemia categories (NGT, GDM and DIP), type 2 diabetes and then ethnicity (Indigenous Australian [49.1\%]; non-Indigenous Australian [32.4\% Europid, 8.4\% Indian, 2.9\% Filipino and
$7.2 \%$ others]). C-peptide and triacylglycerol were $\log _{e}$-transformed prior to analysis and CRP was considered as a dichotomous variable defined as 'detected' or 'not detected' using a manufacturer quoted detection limit of $0.3 \mathrm{mg} / \mathrm{l}(2.9 \mathrm{nmol} / \mathrm{l})$ as the cut-off (the distribution of CRP in our population could not be normalised to allow for analysis as a continuous variable). Means of continuous variables were compared using Student's $t$ test or Wilcoxon rank sum test according to distribution. Prevalence of categorical variables were compared with Pearson's $\chi^{2}$ test. The neonatal outcomes assessed were LGA, birthweight $z$ score, SSF and percentage of body fat. Each outcome was regressed on each cord blood metabolic marker separately (glucose, HDL-cholesterol, C-peptide, triacylglycerol and CRP $>0.3 \mathrm{mg} / \mathrm{l}$ ) using multivariable (linear for continuous and logistic for categorical outcomes) regression models with adjustment for diabetes type (NGT, GDM/DIP and type 2 diabetes), Indigenous and non-Indigenous ethnicity, baby sex and maternal age. A multivariable model was selected where each outcome was regressed on a fixed base covariate set, which included diabetes type, Indigenous and non-Indigenous ethnicity, baby sex and maternal age (for each additional 5 years), and on a set of covariates with $p$ value $<0.1$ (in the multivariable model), including maternal BMI (for each additional $3 \mathrm{~kg} / \mathrm{m}^{2}$ ), smoking in pregnancy, and simultaneously all the cord blood markers, provided there was no collinearity. Each of these multivariable models were additionally adjusted for maternal BMI, nulliparity and smoking in pregnancy. A sensitivity analysis was undertaken whereby multivariable models were repeated for each of the neonatal outcomes and maternal BMI was replaced with gestational weight gain (GWG), as GWG was only available for a subset of the participants $(n=510)$. Possible interactions between each cord blood metabolic marker and each of ethnicity, diabetes type and sex were assessed by adding in the models a marker by each of ethnicity, diabetes type and sex multiplicative term. If significant, the interaction term was included in the final multivariable model.

Pathway analysis was completed using Stata command medeff on the final multivariable models identified as described above. To assess the 'mediation' effect of each statistically significant cord blood marker on the association between maternal BMI and each neonatal outcome, $\beta$ coefficients (continuous outcomes) and ORs (binary outcomes) for the 'total' effect (effect of maternal BMI on neonatal weight/adiposity with all covariates excluding the mediator) and 'direct' effect (effect of maternal BMI on neonatal weight/adiposity with all covariates including mediator) were generated.

\section{Results}

A total of 645 mother-infant pairs with data on cord blood measures were included. Table 1 displays the maternal and infant characteristics by diabetes type and ethnicity. Among women 


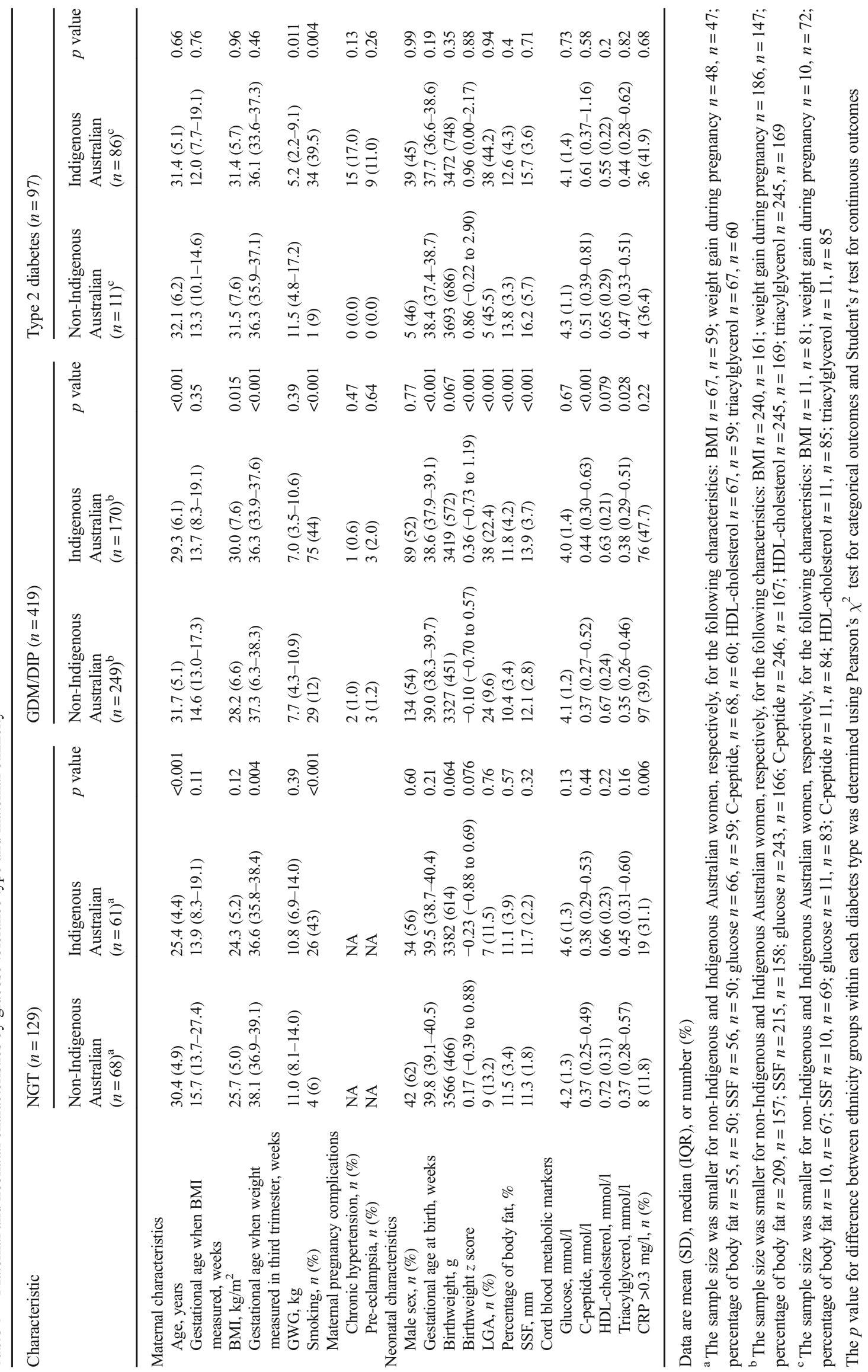


with GDM/DIP, Indigenous Australian women were younger (Indigenous vs non-Indigenous, 29.3 years vs 31.7 years), had a higher BMI $\left(30.0 \mathrm{~kg} / \mathrm{m}^{2}\right.$ vs $\left.28.2 \mathrm{~kg} / \mathrm{m}^{2}\right)$ and were more likely to smoke than non-Indigenous Australian women. Babies of Indigenous Australian women with GDM/DIP also had higher birthweights, birthweight $z$ scores $(0.36$ vs -0.10$)$ and neonatal adiposity (percentage body fat $11.8 \%$ vs $10.4 \%$; SSF $13.9 \mathrm{~mm}$ vs $12.1 \mathrm{~mm})$. Indigenous Australian women with GDM/DIP had higher levels of cord C-peptide $(p<0.001)$ and cord triacylglycerol $(p=0.028)$ than did non-Indigenous Australian women with GDM/DIP.

Among those with NGT, Indigenous Australian women had a higher prevalence of CRP $>0.3 \mathrm{mg} / 1$ than did their nonIndigenous counterparts ( $31 \%$ vs $12 \%$ ). There were no significant differences for cord blood markers between Indigenous and non-Indigenous Australian women with type 2 diabetes.

Table 2 displays the associations between cord blood markers and neonatal outcomes adjusted for diabetes type, ethnicity, baby sex and maternal age. C-peptide had a strong positive association with each outcome (all $p$ values $<0.001$ ). Glucose and triacylglycerol had significant negative associations with birthweight $z$ score, and percentage of body fat. Cord HDL-cholesterol and CRP were not significantly associated with the neonatal adiposity outcomes.

Figure 1a displays the multivariable relationships between cord blood markers, each covariate and birthweight $z$ score. Greater cord glucose was significantly associated with lower birthweight $z$ score. There was no significant interaction between glucose and ethnicity for outcome of birthweight $z$ score. Significant interactions were observed between ethnicity and triacylglycerol, showing a significant negative association for Indigenous but not non-Indigenous Australian women, and between ethnicity and C-peptide $(p=0.004)$, showing a much stronger positive effect in Indigenous than in non-Indigenous Australian women. C-peptide remained strongly and positively associated with the outcome of SSF (Fig. 1b), LGA (Fig. 1c) and percentage of body fat (Fig. 1d) after adjusting for known covariates including maternal BMI. Cord glucose was also negatively associated with LGA (Fig. 1c) and percentage of body fat (Fig. 1d).

Multivariable analyses performed on a subpopulation with available information for GWG showed that GWG was not an important confounder for the associations between cord blood markers and neonatal outcomes (data not shown).

On pathway analysis, C-peptide was the strongest mediator of the association between maternal BMI and each neonatal outcome. The mediation analysis estimated that the total effect of maternal BMI $\left(+3 \mathrm{~kg} / \mathrm{m}^{2}\right)$ on LGA was OR $1.27(95 \% \mathrm{CI}$ $1.11,1.45)$ with $18 \%(13,36)$ of this effect mediated by cord Cpeptide. The direct effect of maternal BMI on LGA as estimated from a multivariable logistic model was OR 1.22 (95\% CI 1.10, 1.36) (Fig. 2a). The total effect of maternal BMI $\left(+3 \mathrm{~kg} / \mathrm{m}^{2}\right)$ on percentage of neonatal body fat was $\beta 0.42$ (95\% CI $0.27,0.54)$ with $11 \%(95 \% \mathrm{CI} 8,17)$ of this effect mediated by $\log _{e} \mathrm{C}$ peptide, resulting in a direct effect of $\beta 0.36$ (95\% CI 0.20 , $0.50)$ (Fig. 2b). The total effect of maternal BMI $\left(+3 \mathrm{~kg} / \mathrm{m}^{2}\right)$ on birthweight $z$ score was $\beta 0.13$ (95\% CI $0.1,0.16)$ with $14 \%$ of this effect mediated by $\log _{e} \mathrm{C}$-peptide, resulting in a direct effect of $\beta 0.12$ (95\% CI 0.08, 0.16).

\section{Discussion}

In this prospective birth cohort study involving mothers across the spectrum of hyperglycaemia in pregnancy, we reported three key findings. First, cord C-peptide was strongly associated with neonatal adiposity as measured by birthweight $z$ score, SSF, LGA and neonatal fat. This was after accounting for diabetes type, ethnicity, baby sex, maternal age, gestational age, nulliparity, maternal BMI (or GWG), smoking in pregnancy, cord glucose and triacylglycerol. Second, cord blood triacylglycerol was negatively associated with birthweight $z$ score

Table 2 Associations of cord blood markers with neonatal adiposity outcomes

\begin{tabular}{lllll}
\hline Cord metabolic markers & $\begin{array}{l}\text { Birthweight } z \text { score } \\
\beta(95 \% \mathrm{CI})\end{array}$ & $\begin{array}{l}\text { SSF } \\
\beta(95 \% \mathrm{CI})\end{array}$ & $\begin{array}{l}\text { LGA } \\
\text { OR }(95 \% \mathrm{CI})\end{array}$ & $\begin{array}{l}\text { Percentage of body fat } \\
\beta(95 \% \mathrm{CI})\end{array}$ \\
\hline C-peptide, $\mathrm{nmol} / \mathrm{l}$ & $0.57(0.42,0.71) ;$ & $0.83(0.41,1.25) ;$ & $3.14(2.11,4.68) ;$ & $1.20(0.69,1.71) ;$ \\
& $p<0.001$ & $p<0.001$ & $p<0.001$ & $p<0.001$ \\
Glucose, mmol/1 & $-0.13(-0.20,-0.05) ;$ & $-0.20(-0.41,0.017) ;$ & $0.78(0.65,0.94) ;$ & $-0.43(-0.69,-0.16) ;$ \\
& $p=0.001$ & $p=0.071$ & $p=0.010$ & $p=0.001$ \\
HDL-cholesterol, mmol/1 & $0.10(-0.30,0.49) ;$ & $-0.90(-2.03,0.24) ;$ & $1.37(0.56,3.35) ;$ & $-0.42(-1.82,1.99) ;$ \\
Triacylglycerol, mmol/1 & $p=0.62$ & $p=0.12$ & $p=0.49$ & $p=0.56$ \\
& $-0.43(-0.62,-0.24) ;$ & $-0.41(-0.95,0.13) ;$ & $0.52(0.33,0.83) ;$ & $-0.76(-1.42,-0.097) ;$ \\
CRP $>0.3 \mathrm{mg} / 1$ & $p<0.001$ & $p=0.14$ & $p=0.005$ & $p=0.025$ \\
& $-0.14(-0.35,0.18) ;$ & $0.014(0.53,0.57) ;$ & $0.75(0.48,1.17) ;$ & $0.54(-0.14,1.22) ;$ \\
\hline
\end{tabular}

All reported results are adjusted for diabetes type, ethnicity, baby sex and maternal age 
(significant for Indigenous women only), after adjusting for covariates. Third, an important and novel finding of this study is that, on pathway analysis, cord blood C-peptide mediated approximately $20 \%$ of the contribution of maternal BMI to LGA and $10 \%$ to per cent neonatal fat.

Further to our report of the impact of type 2 diabetes on perinatal outcomes [39], our large prospective birth cohort study found that cord blood C-peptide was the metabolic marker most strongly associated with all four outcomes of neonatal adiposity, independent of diabetes type and ethnicity [40, 41]. The strong association between cord C-peptide and neonatal adiposity was also reported in the HAPO study. Our findings extend those of the HAPO study by including women treated for GDM and women with type 2 diabetes [41]. Of note, there are limited studies on cord blood metabolic markers and neonatal adiposity in indigenous and minority populations at high risk of diabetes, in whom it is particularly important to assess potential mechanistic markers contributing to transgenerational cycles of obesity and type 2 diabetes [22, 23, 42]. A study of normoglycaemic Polynesian women from New Zealand found that cord insulin concentrations were positively correlated with birthweight and skinfold thickness [42]. However, that study

\section{a}

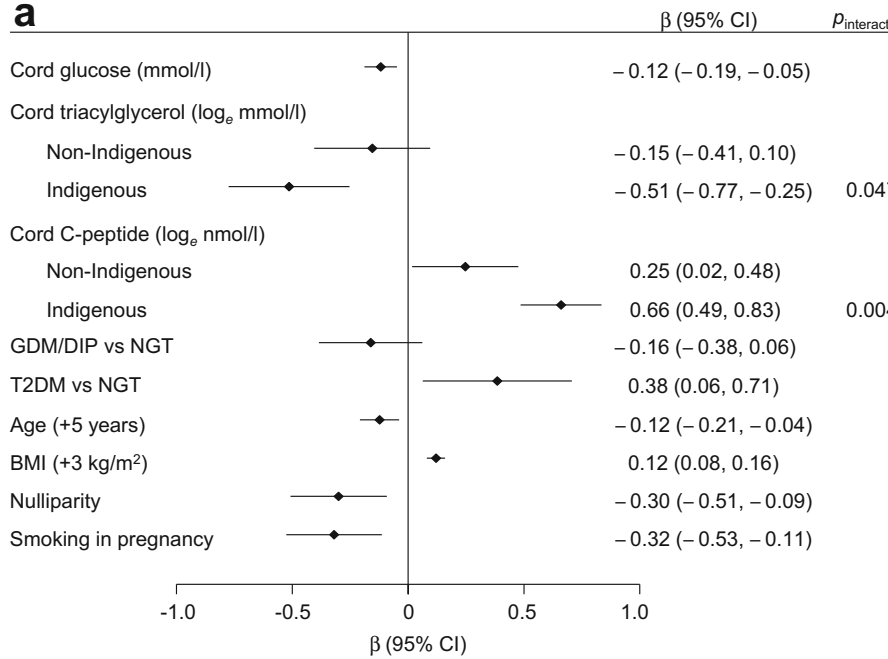

C

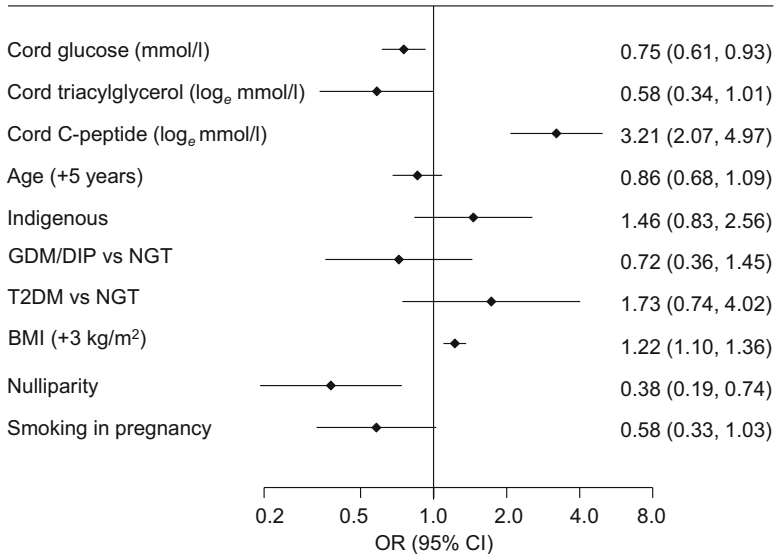

Fig. 1 Multivariable linear regression for the outcomes of birthweight $z$ score (a), SSF (b), LGA (c) and percentage of body fat (d). (a) The model includes significant interaction terms for each of cord C-peptide and triacylglycerol with ethnicity, as well as the main effect of ethnicity. The model includes the following covariates: diabetes type, maternal age (for each additional 5 years), maternal BMI (for each additional $3 \mathrm{~kg}$ / $\mathrm{m}^{2}$ ), smoking in pregnancy, nulliparity, cord glucose, cord triacylglycerol and the interaction with ethnicity, cord C-peptide and the interaction with ethnicity (b) The model includes the following covariates: diabetes type, ethnicity, baby sex, maternal age (for each additional 5 years), nulliparity,
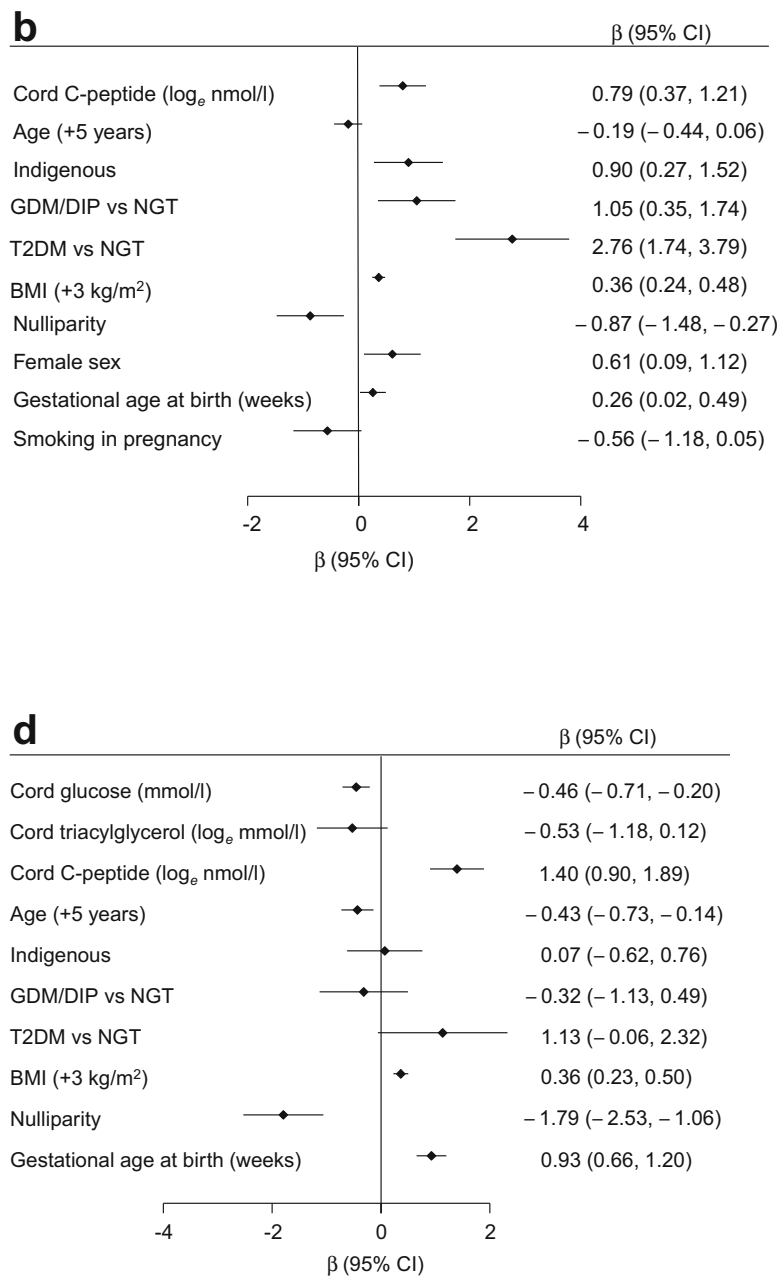

maternal BMI (for each additional $3 \mathrm{~kg} / \mathrm{m}^{2}$ ), smoking in pregnancy, gestational age at birth and cord C-peptide. (c) The model includes the following covariates: diabetes type, ethnicity, maternal age (for each additional 5 years), nulliparity, maternal BMI (for each additional $3 \mathrm{~kg} / \mathrm{m}^{2}$ ), smoking in pregnancy, cord glucose, cord triacylglycerol and cord C-peptide. (d) The model includes the following covariates: diabetes type, ethnicity, maternal age (for each additional 5 years), nulliparity, maternal BMI (for each additional $3 \mathrm{~kg} / \mathrm{m}^{2}$ ), gestational age at birth, cord glucose, cord triacylglycerol and cord C-peptide. T2DM, type 2 diabetes 
did not adjust for potential confounders such as maternal weight and GWG. Hence, our findings are novel for this unique cohort of women with a high proportion of type 2 diabetes in pregnancy, with a multivariable analysis adjusting for several important maternal characteristics.

Interestingly, our study found that cord blood triacylglycerol was inversely associated with birthweight $z$ score; this finding was significant in Indigenous Australian women but not in non-Indigenous women. This inverse relationship has been previously described [11, 43, 44]. Schaefer-Graf et al hypothesised that it was due to reduced lipoprotein lipase (LPL) activity; the role of LPL is to hydrolyse triacylglycerol to NEFA so that the NEFA can be taken up to be stored in adipose tissue [11]. Infants born with LGA have low triacylglycerol levels due to enhanced LPL activity contributing to their increased adipose mass [5]. It has been reported recently that placental LPL activity is positively associated with newborn adiposity [45]. This relationship between fetal triacylglycerol and birthweight $z$ score only reached significance for Indigenous Australian women in our study. Ethnic differences in lipid metabolism have been described both in the pregnant state and for different pregnancy outcomes [46].

Our study also demonstrated that cord blood glucose was inversely associated with birthweight $z$ score, LGA and neonatal body fat. Maternal glucose in pregnancy has consistently been shown to have a continuous effect on neonatal adiposity [47-49] but the relationship between cord glucose and neonatal adiposity has not been as clear [11]. We hypothesise that the strong positive relationship between cord blood C-peptide and neonatal adiposity is likely driving the negative relationship between cord blood glucose and neonatal adiposity observed, as C-peptide enhances metabolism of glucose resulting in lower cord blood glucose levels.

We demonstrated that cord blood C-peptide mediated 20\% of the contribution of maternal BMI to LGA, $14 \%$ to birthweight $z$ score and $10 \%$ to per cent neonatal fat. Cpeptide was not a significant mediator of the relationship between maternal BMI and SSF. Our findings are consistent with The Healthy Start Study of ethnically diverse US women (with normoglycaemia or GDM but not type 2 diabetes), where maternal insulin resistance (HOMA-IR) and glucose levels together accounted for $21 \%$ of the total effect of maternal BMI on neonatal per cent fat mass [48]. Our study extends the current body of evidence by exploring the contribution of fetal C-peptide to the relationship between maternal BMI and neonatal adiposity in a high-risk population including a large number of women with type 2 diabetes. Future research is warranted to examine other steps in the fuel-mediated pathway such as epigenetic modifications and the long-term impact of the metabolic contribution of the in utero environment, particularly in populations at high risk for obesity and type 2 diabetes.
The strengths of this prospective study are that it includes a unique cohort of over 600 women including Indigenous Australians, it is the largest study of women with type 2 diabetes and its analyses adjusted for key variables such as diabetes type, indigenous ethnicity, baby sex, maternal age, maternal BMI and smoking in pregnancy. This is also the largest study of indigenous women internationally with cord blood data and detailed maternal and neonatal characteristics and measurements.

Our study is limited by several factors. First, we did not collect maternal blood samples in pregnancy and therefore could not assess the correlation between maternal and cord metabolic markers. Schaefer-Graf et al have shown that maternal levels of triacylglycerol taken close to delivery correlated significantly with those in cord blood [11], and other studies have reported that maternal $\mathrm{HbA}_{1 \mathrm{c}}$ at the end of pregnancy was positively correlated with neonatal adiposity and cord blood insulin [23]. Therefore, our analyses may not have fully adjusted for the confounding effects of maternal pathology and this may have led to overestimation of the relationship between cord blood markers and neonatal adiposity
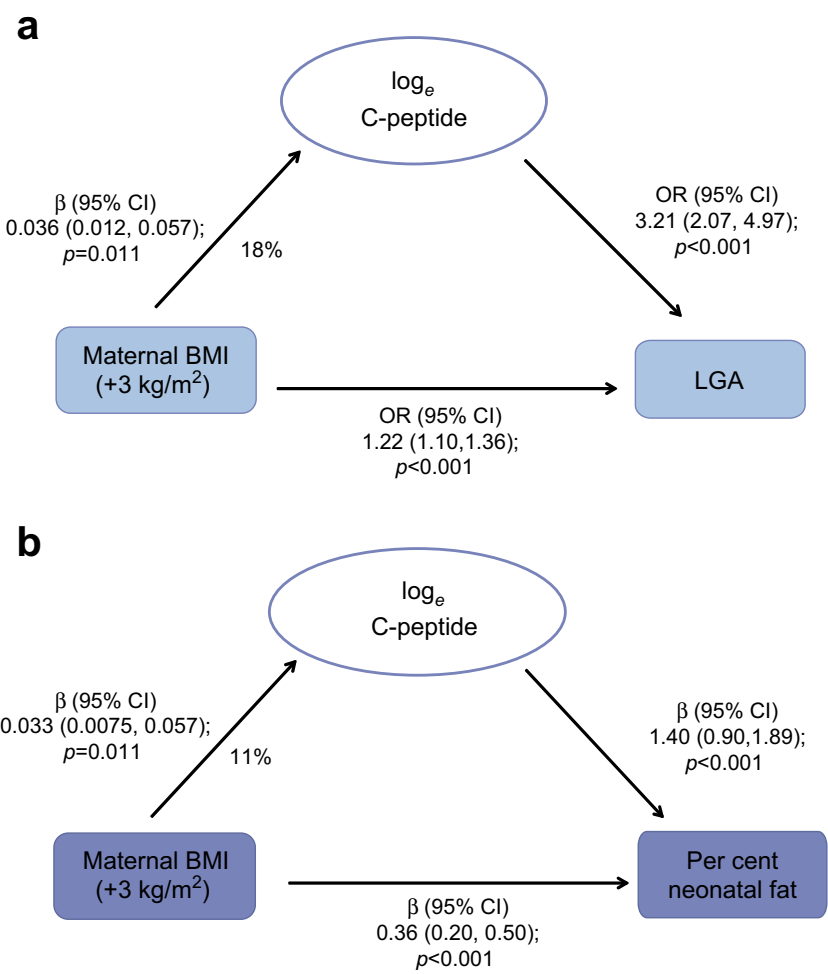

Fig. 2 (a) Pathway analysis of $\log _{e}$ cord C-peptide on the effect of maternal BMI on LGA. Covariates were: diabetes type, ethnicity, maternal age (for each additional 5 years), nulliparity, maternal BMI (for each additional $3 \mathrm{~kg} / \mathrm{m}^{2}$ ), smoking in pregnancy, cord glucose, cord triacylglycerol and cord C-peptide (the same multivariable model as shown in Fig. 1c). (b) Pathway analysis of $\log _{e}$ C-peptide on the effect of maternal BMI on percentage of neonatal fat. Covariates were: diabetes type, ethnicity, maternal age (for each additional 5 years), nulliparity, maternal BMI (for each additional $3 \mathrm{~kg} / \mathrm{m}^{2}$ ), gestational age at birth, cord glucose, cord triacylglycerol and cord C-peptide (the same multivariable model as shown in Fig. 1d) 
outcomes in our study. Second, the clustering of type 2 diabetes and Indigenous Australian ethnicity in our study cohort may have limited our ability to fully explore the interactions of ethnicity as opposed to type 2 diabetes on the associations between cord blood markers and neonatal adiposity. Thus, the stronger associations observed for triacylglycerol and Cpeptide with birthweight $z$ score among Indigenous Australian participants may have also been driven by maternal type 2 diabetes. Third, cord markers in this study were based on venous blood, which may not fully provide a measure of fetal metabolism. Other studies have measured arterial cord blood in order to better measure fetal metabolism. Future studies comparing markers in both venous and arterial blood may be required to provide a deeper understanding of placental transfer mechanisms.

The public health and clinical implications of our findings are to provide further evidence in support of interventions focused on maternal weight. Our findings support the importance of population health interventions to reduce maternal BMI pre-pregnancy, and to limit GWG, in order to improve neonatal adiposity outcomes, among women with and without type 2 diabetes.

In conclusion, high cord blood C-peptide was a strong mediator of the effect of maternal body size on fetal growth in pregnancies across the glucose tolerance spectrum of NGT, GDM and type 2 diabetes. We are uniquely placed to further inform the significance of our findings regarding the role of the intrauterine metabolic environment on the child's future cardiometabolic risk, with planned longitudinal follow-up of this high-risk cohort. Our work will inform the design of future interventions to prevent or reduce the impact of the intergenerational cycle of chronic disease risk in the highrisk Indigenous Australian population, including the very high-risk group of children born to mothers with preexisting type 2 diabetes in pregnancy.

Acknowledgements We gratefully acknowledge all PANDORA study staff and participants, including: C. Whitbread (Wellbeing and Preventable Chronic Disease Division, Menzies School of Health Research, Australia), S. Svenson (Aboriginal Health Domain, Baker Heart and Diabetes Institute, Australia), S. Graham (Wellbeing and Preventable Chronic Disease Division, Menzies School of Health Research, Australia), A. Simmonds (Wellbeing and Preventable Chronic Disease Division, Menzies School of Health Research, Australia), P. Van Dokkum (Aboriginal Health Domain, Baker Heart and Diabetes Institute, Australia), J. Kelaart (Aboriginal Health Domain, Baker Heart and Diabetes Institute, Australia), L. Wood (Wellbeing and Preventable Chronic Disease Division, Menzies School of Health Research, Australia) and L. Davis (Wellbeing and Preventable Chronic Disease Division, Menzies School of Health Research, Australia), as well as Diabetes across the Lifecourse: Northern Australia Partnership investigators, partners, staff, Indigenous Reference Group and Clinical Reference Group, Northern Territory (NT) health professionals from NT Department of Health hospitals, remote primary healthcare, Healthy Living NT and Aboriginal Community Controlled Health Organizations. Diabetes across the Lifecourse: Northern Australia Partnership investigators not named as authors are E. Moore
(Public Health Unit, Aboriginal Medical Services Alliance of Northern Territory, Australia), S. Thomas (Department of Obstetrics and Gynaecology, Royal Darwin Hospital, Australia), S. Eades (Clinical and Population Health, Baker Heart and Diabetes Institute, Australia), S. Corpus (Clinical Services, Danila Dilba Health Service, Australia) and S. Chitturi (Division of Medicine, Royal Darwin Hospital, Australia). The views expressed in this publication are those of the authors and do not reflect the views of the National Health and Medical Research Council of Australia. The funders had no role in study design, data collection and analysis, decision to publish or preparation of the manuscript.

Data availability Data are available on request to the Partnership Steering Committee. They are not attached or on an online repository.

Funding This work was supported by the National Health and Medical Research Council of Australia (NHMRC Partnership Project Grant no. 1032116, NHMRC no. 1078333). Additional support (including pilot funding) was received from NHMRC Program Grant no. 631947. LMB was supported by NHMRC Fellowship no. 605837 and NHMRC Practitioner Fellowship no. 1078477. IL was supported by an Australian Postgraduate award and Menzies scholarship. ELMB was supported by a Heart Foundation post-doctoral fellowship no. 101291. JAB was supported by NHMRC Career Development Fellowship. JES was supported by NHMRC Fellowship no. 1079438. AB was supported by a Viertel Senior Medical Research Fellowship and an NHMRC Senior Research Fellowship no. 1137563.

Duality of interest The authors declare that there is no duality of interest associated with this manuscript.

Contribution statement IL conducted the literature review and drafted the manuscript, with assistance from LMB, ELMB and FB. LMB and AB initiated the study concept, design and partnership. LMB led all aspects of the study including design of the study protocol for funding and ethics applications, supervision of data collection, study conduct, data management and data analysis. IL, DL, MK and VH contributed to data collection and MK contributed additionally to project management. FB performed the statistical analysis and prepared all tables and figures. ELMB assisted with analysis and interpretation of data and revised the article critically for important intellectual content. CC, JAB, KOD, PZ, $\mathrm{JO}, \mathrm{HDM}, \mathrm{AB}$ and JES provided substantial contributions to conception and design of the study and revising the article critically for important intellectual content. ML and ZL contributed intellectually to laboratory methodology and supervised laboratory analyses of cord blood samples. All authors were involved in revising the manuscript for important intellectual content and read and approved the final manuscript. LMB is the guarantor of this work.

\section{References}

1. Murphy HR, Steel SA, Roland JM et al (2011) Obstetric and perinatal outcomes in pregnancies complicated by type 1 and type 2 diabetes: influences of glycaemic control, obesity and social disadvantage. Diabet Med 28(9):1060-1067

2. Hewapathirana NM, Murphy HR (2014) Perinatal outcomes in type 2 diabetes. Curr Diab Rep 14(2):461. https://doi.org/10.1007/ s11892-013-0461-1

3. Feig DS, Palda VA (2002) Type 2 diabetes in pregnancy: a growing concern. Lancet 359(9318):1690-1692

4. McElduff A, Ross GP, Lagström JA et al (2005) Pregestational diabetes and pregnancy an Australian experience. Diabetes Care 28(5):1260-1261. https://doi.org/10.2337/diacare.28.5.1260 
5. Balsells M, Garcia-Patterson A, Gich I, Corcoy R (2009) Maternal and fetal outcome in women with type 2 versus type 1 diabetes mellitus: a systematic review and metaanalysis. J Clin Endocrinol Metab 94(11):4284-4291

6. Colstrup M, Mathiesen ER, Damm P, Jensen DM, Ringholm L (2013) Pregnancy in women with type 1 diabetes: have the goals of St. Vincent declaration been met concerning foetal and neonatal complications? J Matern Fetal Neonatal Med 26(17):1682-1686. https://doi.org/10.3109/14767058.2013.794214

7. Crowther CA, Hiller JE, Moss JR, McPhee AJ, Jeffries WS, Robinson JS (2005) Effect of treatment of gestational diabetes mellitus on pregnancy outcomes. N Engl J Med 352(24):24772486. https://doi.org/10.1056/NEJMoa042973

8. Landon MB, Spong CY, Thom E et al (2009) A multicenter, randomized trial of treatment for mild gestational diabetes. $\mathrm{N}$ Engl J Med 361(14):1339-1348. https://doi.org/10.1056/ NEJMoa0902430

9. Desoye G, Nolan CJ (2016) The fetal glucose steal: an underappreciated phenomenon in diabetic pregnancy. Diabetologia 59(6): 1089-1094

10. Pedersen J (1952) Course of diabetes during pregnancy. Acta Endocrinol 9(4):342-364. https://doi.org/10.1530/acta.0.0090342

11. Schaefer-Graf UM, Graf K, Kulbacka I et al (2008) Maternal lipids as strong determinants of fetal environment and growth in pregnancies with gestational diabetes mellitus. Diabetes Care 31(9):18581863. https://doi.org/10.2337/dc08-0039

12. Barrett HL, Nitert MD, Jones L et al (2013) Determinants of maternal triglycerides in women with gestational diabetes mellitus in the Metformin in Gestational Diabetes (MiG) study. Diabetes Care 36(7):1941-1946. https://doi.org/10.2337/dc12-2132

13. Christou H, Connors JM, Ziotopoulou M et al (2001) Cord blood leptin and insulin-like growth factor levels are independent predictors of fetal growth. J Clin Endocrinol Metab 86(2):935-938. https://doi.org/10.1210/jcem.86.2.7217

14. Karakosta P, Chatzi L, Plana E, Margioris A, Castanas E, Kogevinas M (2011) Leptin levels in cord blood and anthropometric measures at birth: a systematic review and meta-analysis. Paediatr Perinat Epidemiol 25(2):150-163. https://doi.org/10. 1111/j.1365-3016.2010.01163.x

15. Dabelea D, Crume T (2011) Maternal environment and the transgenerational cycle of obesity and diabetes. Diabetes 60(7): 1849-1855. https://doi.org/10.2337/db11-0400

16. Crume TL, Ogden L, Daniels S, Hamman RF, Norris JM, Dabelea D (2011) The impact of in utero exposure to diabetes on childhood body mass index growth trajectories: the EPOCH study. J Pediatr 158(6):941-946

17. Lowe LP, Metzger BE, Lowe WL Jr, Dyer AR, McDade TW, McIntyre HD (2010) Inflammatory mediators and glucose in pregnancy: results from a subset of the Hyperglycemia and Adverse Pregnancy Outcome (HAPO) study. J Clin Endocrinol Metab 95(12):5427-5434. https://doi.org/10.1210/jc.2010-1662

18. Donnelly JM, Lindsay KL, Walsh JM, Horan M, Molloy EJ, McAuliffe FM (2015) Fetal metabolic influences of neonatal anthropometry and adiposity. BMC Pediatr 15:175

19. Dubé M-C, Morisset A-S, Tchernof A, Weisnagel S (2012) Cord blood C-peptide levels relate to the metabolic profile of women with and without gestational diabetes. Acta Obstet Gynecol Scand 91(12):1469-1473

20. Walsh JM, Mahony R, Byrne J, Foley M, McAuliffe FM (2011) The association of maternal and fetal glucose homeostasis with fetal adiposity and birthweight. Eur J Obstet Gynecol Reprod Biol 159(2):338-341

21. Geraghty AA, Alberdi G, O'Sullivan EJ et al (2016) Maternal blood lipid profile during pregnancy and associations with child adiposity: findings from the ROLO study. PLoS One 11(8):e0161206. https:// doi.org/10.1371/journal.pone.0161206
22. Teague AM, Fields DA, Aston CE, Short KR, Lyons TJ, Chernausek SD (2015) Cord blood adipokines, neonatal anthropometrics and postnatal growth in offspring of Hispanic and Native American women with diabetes mellitus. Reprod Biol Endocrinol 13:68. https://doi.org/10.1186/s12958-015-0061-9

23. Westgate JA, Lindsay RS, Beattie J et al (2006) Hyperinsulinemia in cord blood in mothers with type 2 diabetes and gestational diabetes mellitus in New Zealand. Diabetes Care 29(6):1345-1350. https://doi.org/10.2337/dc05-1677

24. Australian Institute of Health Welfare (2010) Diabetes in pregnancy: its impact on Australian women and their babies: Diabetes Series no. 14. Australian Institute of Health and Welfare, Canberra

25. Lee IL, Purbrick B, Barzi F et al (2018) Cohort profile: the Pregnancy and Neonatal Diabetes Outcomes in Remote Australia (PANDORA) study. Int J Epidemiol 47(4):1045-1046. https://doi. org/10.1093/ije/dyy046

26. Longmore DK, Barr ELM, Lee IL et al (2019) Maternal body mass index, excess gestational weight gain, and diabetes are positively associated with neonatal adiposity in the Pregnancy and Neonatal Diabetes Outcomes in Remote Australia (PANDORA) study. Pediatr Obes 14:e12490

27. Kirkham R, Boyle J, Whitbread C et al (2017) Health service changes to address diabetes in pregnancy in a complex setting: perspectives of health professionals. BMC Health Serv Res 17(1): 524. https://doi.org/10.1186/s12913-017-2478-7

28. Kirkham R, Whitbread C, Connors C et al (2017) Implementation of a diabetes in pregnancy clinical register in a complex setting: findings from a process evaluation. PLoS One 12(8):e0179487

29. Hoffman L, Nolan C, Wilson JD, Oats JJ, Simmons D (1998) Gestational diabetes mellitus-management guidelines. The Australasian Diabetes in Pregnancy Society. Med J Aust 169(2): 93-97

30. Metzger BE, Gabbe SG, Persson B et al (2010) International Association of Diabetes and Pregnancy Study Groups recommendations on the diagnosis and classification of hyperglycemia in pregnancy. Diabetes Care 33(3):676-682. https://doi.org/10.2337/ dc09-1848

31. World Health Organisation (2013) diagnostic criteria and classification of hyperglycaemia first detected in pregnancy. Available from https://apps.who.int/iris/bitstream/handle/10665/85975/ WHO_NMH_MND_13.2_eng.pdf?sequence $=1$. Accessed 18 Oct 2019

32. Brown MA, Magee LA, Kenny LC et al (2018) Hypertensive disorders of pregnancy: ISSHP classification, diagnosis, and management recommendations for international practice. Hypertension 72(1):24-43

33. Dobbins TA, Sullivan EA, Roberts CL, Simpson JM (2012) Australian national birthweight percentiles by sex and gestational age, 1998-2007. Med J Aust 197(5):291-294. https://doi.org/10. 5694/mja11.11331

34. Catalano PM, Thomas A, Huston-Presley L, Amini SB (2003) Increased fetal adiposity: a very sensitive marker of abnormal in utero development. Am J Obstet Gynecol 189(6):1698-1704. https://doi.org/10.1016/s0002-9378(03)00828-7

35. Catalano PM, Thomas AJ, Avallone DA, Amini SB (1995) Anthropometric estimation of neonatal body composition. Am J Obstet Gynecol 173(4):1176-1181

36. HAPO Study Cooperative Research Group (2009) Hyperglycemia and Adverse Pregnancy Outcome (HAPO) study: associations with neonatal anthropometrics. Diabetes 58(2):453-459

37. HAPO Study Cooperative Research Group, Metzger BE, Lowe LP et al (2008) Hyperglycemia and adverse pregnancy outcomes. N Engl J Med 358(19):1991-2002. https://doi.org/10.1056/ NEJMoa0707943

38. Cauble JS, Dewi M, Hull HR (2017) Validity of anthropometric equations to estimate infant fat mass at birth and in early infancy. 
BMC Pediatr 17(1):88-88. https://doi.org/10.1186/s12887-0170844-6

39. Maple-Brown L, Lee IL, Longmore D et al (2018) Pregnancy and Neonatal Diabetes Outcomes in Remote Australia: the PANDORA study - an observational birth cohort. Int J Epidemiol 48(1):307318

40. Schwartz R, Gruppuso PA, Petzold K, Brambilla D, Hiilesmaa V, Teramo KA (1994) Hyperinsulinemia and macrosomia in the fetus of the diabetic mother. Diabetes Care 17(7):640-648. https://doi. org/10.2337/diacare.17.7.640

41. HAPO Study Cooperative Research Group (2002) The Hyperglycemia and Adverse Pregnancy Outcome (HAPO) study. Int J Gynaecol Obstet 78(1):69-77

42. Simmons D, Brier BH (2000) Do Polynesians have obesity-driven fuel-mediated teratogenesis? Diabetes Care 23(12):1855-1857. https://doi.org/10.2337/diacare.23.12.1855a

43. Rodie VA, Caslake MJ, Stewart F et al (2004) Fetal cord plasma lipoprotein status in uncomplicated human pregnancies and in pregnancies complicated by pre-eclampsia and intrauterine growth restriction. Atherosclerosis 176(1):181-187. https://doi.org/10. 1016/j.atherosclerosis.2004.04.026

44. Merzouk H, Meghelli-Bouchenak M, el-Korso N, Belleville J, Prost J (1998) Low birth weight at term impairs cord serum lipoprotein compositions and concentrations. Eur J Pediatr 157(4):321-326

45. Heerwagen MJ, Gumina DL, Hernandez TL et al (2018) Placental lipoprotein lipase activity is positively associated with newborn adiposity. Placenta 64:53-60. https://doi.org/10.1016/j.placenta. 2018.03.001

46. Nolan CJ, Riley SF, Sheedy MT, Walstab JE, Beischer NA (1995) Maternal serum triglyceride, glucose tolerance, and neonatal birth weight ratio in pregnancy: a study within a racially heterogeneous population. Diabetes Care 18(12):1550-1556. https://doi.org/10. 2337/diacare.18.12.1550

47. Lowe LP, Metzger BE, Dyer AR et al (2012) Hyperglycemia and Adverse Pregnancy Outcome (HAPO) study: associations of maternal A1C and glucose with pregnancy outcomes. Diabetes Care 35(3):574-580. https://doi.org/10.2337/dc11-1687

48. Shapiro AL, Schmiege SJ, Brinton JT et al (2015) Testing the fuelmediated hypothesis: maternal insulin resistance and glucose mediate the association between maternal and neonatal adiposity, the Healthy Start study. Diabetologia 58(5):937-941. https://doi.org/ 10.1007/s00125-015-3505-z

49. Aris IM, Soh SE, Tint MT et al (2014) Effect of maternal glycemia on neonatal adiposity in a multiethnic Asian birth cohort. The Journal of Clinical Endocrinology \& Metabolism 99(1):240-247. https://doi.org/10.1210/jc.2013-2738

Publisher's note Springer Nature remains neutral with regard to jurisdictional claims in published maps and institutional affiliations. 